\title{
Sapphire and Other Dielectric Waveguide Devices
}

\author{
Markus Pollnau \\ Integrated Optical Micro Systems Group, MESA+ Institute for Nanotechnology, \\ University of Twente, P.O. Box 217, NL-7500 AE Enschede, The Netherlands
}

\section{Introduction}

In recent years, the field of integrated optics has made remarkable progress. Optical gain and high output power can be obtained in rare-earth or transition-metal ion doped dielectric materials. These robust materials also offer high optical damage thresholds. As miniaturized waveguide lasers and amplifiers, these systems will find applications whenever there is a need for on-chip optical gain in combination with high optical power, wavelength diversity, ultra-short pulses, or second- and third-order optical nonlinearities which can be exploited in order to integrate various optical functions on a chip. This paper reviews our and our colleagues' recent results concerning waveguide fabrication [1] and channel waveguide lasers in $\alpha-\mathrm{Al}_{2} \mathrm{O}_{3}: \mathrm{Ti}^{3+}$ (hereafter abbreviated as Ti:sapphire) near $800 \mathrm{~nm}$ [2].

\section{Proton-implanted Ti:sapphire channel waveguide lasers}

Transition-metal-ion-doped materials exhibiting $3 d \leftrightarrow 3 d$ electronic transitions are useful as broadly tunable laser sources and for the generation of ultrashort pulses, due to strong homogeneous broadening of the gain bandwidth by interaction with lattice vibrations. Ti:sapphire allows continuous-wave tunability from $\sim 670-1100 \mathrm{~nm}$ and the generation of pulses as short as a few fs. Although laser thresholds as low as $\sim 100 \mathrm{~mW}$ have been demonstrated [3], in general thresholds are much higher. This has prompted work on creating Ti:sapphire waveguides in order to lower the laser threshold. In addition, the waveguide geometry offers the opportunity of integrating devices with the laser for tuning or mode-locking for a high-repetition-rate, compact femtosecond-laser source [4], and of integrating with other waveguide circuits, e.g. for the purposes of a sensing device that utilizes the large Ti:sapphire bandwidth.

Ion beam implantation was used for the fabrication of waveguide laser structures in insulators. For light ions, electronic damage is comparatively low; at lower energies near the end of the ions' track nuclear collisions predominate, leading to the displacement of lattice ions and resulting typically in a large cascade of damages. As a consequence, a (partial) amorphization of the material appears at the end of the ions' track, with a positive or negative refractive-index change depending on the ion fluence, implanted material, and nature of the interactions.

Buried channel waveguides were produced by proton implantation in sapphire [5] and Ti:sapphire bulk crystals [6]. Proton irradiation in sapphire or Ti:sapphire results in a negative refractive-index change of the implanted zone. Therefore, buried channel waveguides were fabricated by implanting regions of low refractive index surrounding the intended channel waveguide region. Implantations were performed with high energy protons of $0.5-1 \mathrm{MeV}$. Vertical confinement was provided with a lower and upper barrier resulting from uniform irradiations of the whole sample surface at different energies. Within this buried planar waveguide, horizontal confinement was obtained by successive irradiations through a slit on both sides of the intended channel. A better lateral confinement is achieved by a vertically uniform distribution of the refractive-index change within the side barriers. Therefore, irradiations with several angles of incidence were performed, resulting in vertically stacked low-refractive-index barriers [6].

Refractive-index changes of the planar waveguide parts were characterized by m-lines spectroscopy. Only negative refractive-index changes were observed in proton-irradiated sapphire, with gradient shape and maximum refractive-index decrease of typically $0.5-1 \%$, for irradiation with $1-\mathrm{MeV}$ protons at a fluence of $10^{16} \mathrm{H}^{+} / \mathrm{cm}^{2}$. The damaged barriers were localized $\sim 8-\mu \mathrm{m}$ deep for $1-\mathrm{MeV}$ protons, which is in good accordance with Monte-Carlo calculations of the probability of a target atom being displaced from its lattice site (TRIM calculations). Proton implantation induces well-controlled refractive-index decreases, hence the guided regions are formed by writing low-refractive-index barriers around the intended guided regions and their mode profiles can be easily adjusted by changing the implantation parameters and geometries. By choosing different channel dimensions and optical confinement in the horizontal and vertical directions, we produced elliptical fundamental-mode profiles, with larger modal fields in the horizontal direction. Also cylindrical mode shapes can be achieved by adjusting the implantation energies, doses, and geometries. Buried channel waveguides showed losses of 1.0-1.3 dB/cm at $720 \mathrm{~nm}$. The higher loss level of the narrower guides was due to the stronger interaction of the modal field with the optical barriers. 
Lasing experiments [6] with the Ti:sapphire buried channel waveguides were performed with an $\mathrm{Ar}^{+}$pump laser. The laser resonator was formed by attaching at the end-faces two thin dielectric mirrors with high reflectivity and transmission at the lasing and pump wavelength, respectively. $\mathrm{CW}$ laser operation was obtained in channel waveguides with a height of $5 \mu \mathrm{m}$ and widths of 10 and $15 \mu \mathrm{m}$ at absorbed pump power thresholds of 230 and 260 $\mathrm{mW}$, respectively. $\pi$-polarized lasing was observed near $780 \mathrm{~nm}$. Near-diffraction-limited laser emission was obtained from the 10- $\mu \mathrm{m}$-wide channels with values of $\mathrm{M}_{\mathrm{x}}{ }^{2}=1.5$ and $\mathrm{M}_{\mathrm{y}}{ }^{2}=1.2$ for the horizontal and perpendicular directions, respectively. By replacing the HR output mirror with one having a transmission of $4.6 \%$ at the lasing wavelength the threshold values increased to 260 and $290 \mathrm{~mW}$ for the 10 - and 15 - $\mu \mathrm{m}$-wide channels, respectively. Slope efficiency values of $3 \%$ and $2.2 \%$ were obtained from the 15 - and $10-\mu \mathrm{m}$-wide channels, respectively. The corresponding output powers are 17.5 and $12.4 \mathrm{~mW}$ for $1 \mathrm{~W}$ of absorbed power.

Other methods for fabricating Ti:sapphire channel waveguides include femtosecond laser writing [7], ion indiffusion [8], and pulsed laser deposition [9] in combination with reactive ion etching [10,11]. The latter methods have lead to the demonstration of broadband luminescent emitters [12], which show potential as light sources in optical coherence tomography [13], and planar [14], rib channel [15], and in-diffused channel [16] waveguide lasers.

\section{Conclusions}

Different fabrication methods have been explored successfully and surface and buried channel waveguide lasers have been demonstrated in Ti:sapphire for the first time. Since the propagation losses of these first-generation waveguides are still rather high, substantial improvement is required in order to obtain ultra-low-threshold waveguide lasers and to explore their full potential as light sources in various applications in integrated optics.

\section{Acknowledgments}

The author thanks A. Crunteanu, L. Laversenne, V. Apostolopoulos, C.N. Borca, P. Hoffmann, R.P. Salathé, C. Grivas, T. Bhutta, T.C. May-Smith, R.W. Eason, D.P. Shepherd, P. Moretti, and J. Mugnier for contributions to the experiments and helpful discussions. This work was partially supported by the Swiss National Science Foundation.

\section{References}

1. M. Pollnau and Y.E. Romanyuk, Optical waveguides in laser crystals, C. R. Phys. 8, 123-137 (2007).

2. M. Pollnau, C. Grivas, L. Laversenne, J.S. Wilkinson, R.W. Eason, and D.P. Shepherd, Ti:sapphire waveguide lasers, Laser Phys. Lett. 4, 560-571 (2007)

3. A.M. Kowalevicz, T.R. Schibli, F.X. Kärtner, and J.G. Fujimoto, Ultralow-threshold Kerr-lens mode-locked Ti: $\mathrm{Al}_{2} \mathrm{O}_{3}$ laser, Opt. Lett. 27, 2037-2039 (2002).

4. B. Stormont, I.G. Cormack, M. Mazilu, C.T.A. Brown, D. Burns, and W. Sibbett, Low-threshold, multi-gigahertz repetition-rate femtosecond Ti:sapphire laser, Electron. Lett. 39, 1820-1822 (2003).

5. L. Laversenne, P. Hoffmann, M. Pollnau, P. Moretti, and J. Mugnier, Designable buried waveguides in sapphire by proton implantation, Appl. Phys. Lett. 85, 5167-5169 (2004).

6. C. Grivas, D.P. Shepherd, R.W. Eason, L. Laversenne, P. Moretti, C.N. Borca, and M. Pollnau, Room-temperature continuous-wave operation of Ti:sapphire buried channel-waveguide lasers fabricated via proton implantation, Opt. Lett. 31, 3450-3452 (2006).

7. V. Apostolopoulos, L. Laversenne, T. Colomb, C. Depeursinge, R.P. Salathé, M. Pollnau, R. Osellame, G. Cerullo, and P. Laporta, Femtosecond-irradiation-induced refractive-index changes and channel waveguiding in bulk $\mathrm{Ti}^{3+}$ :sapphire, Appl. Phys. Lett. 85, 1122-1124 (2004).

8. V. Apostolopoulos, L.M.B. Hickey, D.A. Sager, and J.S. Wilkinson, Gallium-diffused waveguides in sapphire, Opt. Lett. 26, 1586-1588 (2001).

9. A.A. Anderson, R.W. Eason, M. Jelínek, C. Grivas, D. Lane, K. Rogers, L.M.B. Hickey, and C. Fotakis, Growth of Ti:sapphire single crystal thin films by pulsed laser deposition, Thin Solid Films 300, 68-71 (1997).

10. A. Crunteanu, M. Pollnau, G. Jänchen, C. Hibert, P. Hoffmann, R.P. Salathé, R.W. Eason, C. Grivas, and D.P. Shepherd, Ti:sapphire rib channel waveguide fabricated by reactive ion etching of a planar waveguide, Appl. Phys. B 75, 15-17 (2002).

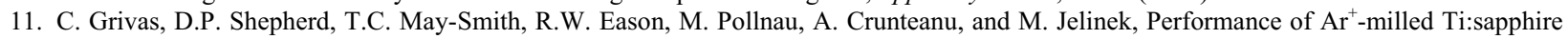
rib waveguides as single transverse mode broadband fluorescence sources, IEEE J. Quantum Electron. 39, 501-507 (2003).

12. M. Pollnau, R.P. Salathé, T. Bhutta, D.P. Shepherd, and R.W. Eason, Continuous-wave broadband emitter based on a transition-metal-iondoped waveguide, Opt. Lett. 26, 283-285 (2001).

13. A.M. Kowalevicz, T. Ko, I. Hartl, J.G. Fujimoto, M. Pollnau, and R.P. Salathé, Ultrahigh resolution optical coherence tomography using a superluminescent light source, Opt. Express 10, 349-353 (2002).

14. A.A. Anderson, R.W. Eason, L.M.B. Hickey, M. Jelínek, C. Grivas, D.S. Gill, and N.A. Vainos, Ti:sapphire planar waveguide laser grown by pulsed laser deposition, Opt. Lett. 22, 1556-1558 (1997).

15. C. Grivas, D.P. Shepherd, T.C. May-Smith, R.W. Eason, and M. Pollnau, Single-transverse-mode Ti:sapphire rib waveguide laser, Opt. Express 13, 210-215 (2005).

16. L.M.B. Hickey, V. Apostolopoulos, R.W. Eason, J.S. Wilkinson, and A.A. Anderson, Diffused Ti:sapphire channel-waveguide lasers, J. Opt Soc. Am. B 21, 1452-1462 (2004). 